\title{
Spermine Inhibition of Monocyte Activation and Inflammation
}

\author{
Minghuang Zhang, ${ }^{1}$ Lyudmila V. Borovikova,' Haichao Wang, ${ }^{1,2}$ \\ Christine Metz, ${ }^{1}$ and Kevin J. Tracey ${ }^{1,3}$ \\ ${ }^{1}$ The Picower Institute for Medical Research, Manhasset, New York, \\ U.S.A. \\ Departments of ${ }^{2}$ Emergency Medicine and ${ }^{3}$ Surgery, North Shore \\ University Hospital, Manhasset, New York, U.S.A.
}

Accepted August 2, 1999.

\begin{abstract}
The innate immune system functions as a defensive front line against pathogenic invasion, but the proinflammatory products of activated monocytes and macrophages (e.g., TNF and NO) can also injure normal cells. Anti-inflammatory mediators restrain the innate immune response and prevent excessive collateral tissue damage. Spermine, a ubiquitous biogenic polyamine, specifically and reversibly suppresses the synthesis of monocyte proinflammatory cytokines. This may provide a counterregulatory mechanism to restrain monocyte activation in injured or infected tissues and in tumors where spermine levels are significantly increased. Here we show that monocyte spermine uptake was signifi-
\end{abstract}

cantly increased following lipopolysaccharide stimulation. The polyamine analogue 1,4-bis(3-aminopropyl)-piperazine (BAP) inhibited LPS-stimulated monocyte spermine uptake via the "nonselective" polyamine transporter. BAP fully restored macrophage TNF synthesis despite the presence of spermine, indicating that the mechanism of monocyte deactivation by spermine is dependent on spermine uptake. Administration of BAP in vivo significantly augmented the development of carrageenan-induced paw edema and nitric oxide release. Thus, endogenous spermine normally inhibits the innate inflammatory response by restraining macrophages.

\section{Introduction}

Monocytes and macrophages occupy a critical role in the orchestration of a competent innate immune response. When activated by pathogenic stimuli or injury, macrophages release tumor necrosis factor (TNF), other cytokines, and nitric oxide (NO) that bolster defense against invasion. NO, for instance, is cytotoxic to invading organisms, and increases regional blood flow to the affected tissues (1), while TNF and interleukin-1 (IL-1) activate endothelial cells, recruit

Address correspondence and reprint requests to: Dr. Minghuang Zhang, The Picower Institute for Medical Research, 350 Community Drive, Fourth Floor, Manhasset, NY 11030, U.S.A. Phone: 516-562-9476, Fax: 516-365-5090; E-mail: mzhang@picower.edu leukocytes, enhance procoagulant activity, and facilitate tissue remodeling $(2,3)$. The importance of these responses is revealed by studies of TNF receptor knock-out mice, which are exquisitely sensitive to infection by intracellular pathogens, reflecting the normal role of TNF to enhance host defenses against infection (4). These beneficial effects, however, are critically dependent on the magnitude of the immune response, because larger amounts of host-derived cytotoxins cause collateral damage to normal tissues $(3,5,6)$. Moreover, when release is sufficient to cause systemic exposure, macrophage-derived cytotoxins mediate lethal shock and tissue injury (7). To prevent these potentially lethal effects, the immune system has evolved a series of counter- 
regulatory mechanisms that inhibit macrophage activation. For example, anti-inflammatory cytokines (IL-10) and TGF- $\beta$ ) and glucocorticoid hormones are released during inflammation and inhibit macrophage release of TNF and NO (8-13). Ultimately, therefore, successful resolution of pathogenic invasion depends on the beneficial activities of macrophage products within the context of a closely regulated macrophage response.

Spermine, a ubiquitous biogenic polyamine, is one of the most abundant molecules in eukaryotes. Proliferating cells and tissues generally have higher levels of polyamines, and injured cells release spermine into the extracellular milieu. Spermine levels increase significantly at sites of infection and injury (14) as well as in tumors (15). More than $\mathbf{4 0}$ years ago, Hirsch and Dubois discovered that local accumulation of spermine in infected tissues can curb the growth of mycobacteria (16). More recently, we found that spermine is a specific and reversible inhibitor of monocyte activation that suppresses the synthesis of TNF and other proinflammatory cytokines by human peripheral blood mononuclear cells. Spermine suppresses proinflammatory cytokine synthesis via a post-transcriptional mechanism (17). Direct application of spermine together with carrageenan, an activator of the innate immune response, attenuates the development of carrageenan-induced inflammation, suggesting that macrophages are sensitive to spermine inhibition in vivo. In agreement with this mechanism, macrophages derived from polyamine-depleted mice (after treatment with a polyamine biosynthesis inhibitor, $\mathrm{DMFMO}_{\mathrm{me}}$ ) release more TNF and IL-1 in response to lipopolysaccharide (LPS) challenge (18). On the basis of these and other results, we reasoned that the macrophage deactivating activity of spermine might be a critical mechanism to restrain macrophages from causing excessive collateral damage. Increased spermine levels at sites of tissue injury would "inform" macrophages that extensive tissue injury had occurred, and curb their further inflammatory activity.

Polyamine transport systems in mammalian cells have been divided functionally into two classes that are distinguished by differential affinity for terminal aminobutyl or aminopropyl groups (19). One transporter class, termed "nonselective," facilitates the uptake of spermine, spermidine, and putrescine; the other class is specific for polyamines with aminopropyl groups (spermine and spermidine) but has a low affinity for putrescine. Cells alter the activity of the polyamine uptake systems to regulate intracellular polyamine availability $(20)$. Whereas active polyamine transport has been demonstrated in macrophages (21), a role for macrophage polyamine transport activity in modulating inflammatory responses has not been described previously.

We now report that human monocytes express a nonselective polyamine transporter, and that LPS stimulation significantly increased monocyte uptake of spermine via this transporter. When spermine accumulation via this transport system was inhibited by treatment with the polyamine analogue 1,4-bis(3-aminopropyl)-piperazine (BAP), monocytes were not deactivated, and their proinflammatory activity was unrestrained despite the presence of elevated extracellular spermine. Application of BAP with carageenan in vivo significantly increased carrageenan-induced local inflammatory responses, indicating that monocytes incorporate endogenous spermine as a normal counterregulatory mechanism to restrain the magnitude of the innate immune response.

\section{Materials and Methods}

\section{Cell Isolation and Culture}

Human leukocyte buffy coats were obtained by leukopheresis of blood from normal individual donors to the Long Island Blood Service (Melville, NY). Human peripheral blood mononuclear cells (PBMCs) were freshly isolated from $30 \mathrm{ml}$ of leukocyte buffy coats by density gradient centrifugation through Ficoll-Paque using a procedure recommended by the vendor (Pharmacia, Uppsala, Sweden), with a yield typically of $2-4 \times 10^{8}$ cells. Cells were resuspended in RPMI 1640 medium (Gibco BRL, Grand Island, NY) containing $10 \%$ heat-inactivated human serum, $0.1 \%$ L-glutamine, and $0.01 \%$ gentamicin, and plated in 96-well plates at $5 \times 10^{5}$ cells per well in $200 \mu \mathrm{l}$, then cultured at $37^{\circ} \mathrm{C}$ in a humidified atmosphere of $5 \% \mathrm{CO}_{2}$ in air. Nonadherent cells were removed after overnight culture, and adherent cells (monocyte cultures) were then used in the experiments.

\section{Polyamine Uptake}

Human monocytes in 96-well plates were stimulated by adding $135 \mu \mathrm{l}$ of medium with 100 $\mathrm{ng} / \mathrm{ml}$ freshly sonicated Escherichia coli endotoxin (LPS, Sigma, St. Louis, MO). After 2 to $24 \mathrm{hr}$ 
stimulation, $15 \mu \mathrm{l}$ of medium with ${ }^{14} \mathrm{C}$-labeled polyamine (Amersham, Arlington Heights, IL) was added to the final concentrations indicated in the absence or presence of BAP (Aldrich, Milwaukee, WI). Following a 25 -min incubation at $4^{\circ} \mathrm{C}$ (for membrane binding) or $37^{\circ} \mathrm{C}$ (for both membrane binding and cellular uptake), media were aspirated, and the cells were rinsed three times with $200 \mu \mathrm{l}$ of phosphate-buffered saline (PBS). Cells were then solubilized in $100 \mu \mathrm{l}$ of 1 $\mathrm{N} \mathrm{NaOH}$, and $80 \mu \mathrm{l}$ of sample was transferred to a 96-well Lumaplate ${ }^{\mathrm{TM}}$ (Packard, Downers Grove, IL). Samples were dried at room temperature overnight, and the cell-associated radioactivity was determined on a Packard liquid scintillation counter. Polyamine uptake is defined as the difference between cell-associated radioactivity at $37^{\circ} \mathrm{C}$ and that at $4^{\circ} \mathrm{C}$.

\section{Cytokine Assay}

To stimulate cytokine production from human monocytes, freshly sonicated LPS was added to a final concentration of $100 \mathrm{ng} / \mathrm{ml}$. To evaluate the effect of BAP on the inhibitory activity of spermine on monocyte TNF synthesis, human monocytes were preincubated with the concentration of BAP indicated for $30-60 \mathrm{~min}$ and $35 \mu \mathrm{M}$ of spermine (Sigma) for another 30-60 min, then stimulated with $100 \mathrm{ng} / \mathrm{ml}$ of LPS. After $4 \mathrm{hr}$ of LPS stimulation, supernatants were harvested for cytokine assay. TNF levels in the supernatants were determined by ELISA. Briefly, serial dilution of recombinant human TNF was used to generate standard curves. The 96-well microtiter plates coated with monoclonal anti-TNF antibodies were incubated with $60 \mu \mathrm{l}$ of standards or supernatant samples for $2 \mathrm{hr}$ at room temperature or overnight at $4^{\circ} \mathrm{C}$. After washing with buffer containing $20 \mathrm{mM}$ Tris- $\mathrm{HCl}, \mathrm{pH} 7.4,150$ $\mathrm{mM} \mathrm{NaCl}$, and $0.05 \%$ Tween-20, $60 \mu \mathrm{l}$ of polyclonal anti-TNF antibodies diluted $(1: 200)$ in dilution buffer ( $10 \mathrm{mM}$ Tris- $\mathrm{HCl}, \mathrm{pH} 7.4,150 \mathrm{mM}$ $\mathrm{NaCl}, 0.2 \%$ Tween-20, and $1 \%$ goat serum) was added and incubated at room temperature for 2 hr. Following several washes to remove the unbound antibodies, the bound antibodies were reacted for 30 min with alkaline phosphatase-conjugated goat anti-rabbit IgG $(\mathrm{H}+\mathrm{L})$ (Boehringer Mannheim, Indianapolis, IN) diluted 1:2500 in dilution buffer. After washing, the amounts of bound second antibodies were then determined by assaying for alkaline phosphatase activity with $100 \mu$ l of freshly prepared p-nitrophenylphosphate in diethanolamine buffer $(10 \mathrm{mM}$ diethanolamine and $0.5 \mathrm{mM} \mathrm{MgCl}, \mathrm{pH} 9.5)$. After $30 \mathrm{~min}$ incubation at room temperature, the absorption at $405 \mathrm{~nm}$ was determined using a microtitre plate reader for TNF levels.

\section{Carrageenan-Induced Footpad Inflammation}

Paw edema was induced by injecting $100 \mu \mathrm{l}$ of $0.2 \% \lambda$-carrageenan (Sigma) in PBS, $\mathrm{pH} 7.4$, into the plantar surface of the left hindpaw of male Lewis rats (300-350 g). Spermine and/or BAP at the concentrations indicated were coadministrated with carrageenan. The right hindpaw was injected with $100 \mu \mathrm{l}$ of PBS as a control. The thickness of the carrageenan-treated and PBStreated hindpaw was measured with calipers at the time points indicated, and the difference was used as an index of inflammation. To recover edema fluid from rat paws, rats were sacrificed 16-24 hr after carrageenan administration. Paws were excised at the level of the calcaneus bone, and placed in a 6-ml Falcon 2063 tube (a 4- to 4.5-mm hole was made with the cut side down). The 6-ml Falcon tube was then placed in a $15-\mathrm{ml}$ Falcon 2097 conical tube with $50 \mu \mathrm{l}$ of PBS and centrifuged at 250-300 $\times g$ for $20 \mathrm{~min}$ and the resultant fluid was recovered for assay as described.

\section{Determination of NO Production in Rat Paw}

NO production in the edema fluid from each paw was determined by measuring nitrite/nitrate level with a nonenzymatic NO assay kit (OXIS, Portland, OR). Serial dilution of nitrite was used to generate standard curves. Briefly, $40 \mu \mathrm{l}$ of sample was adjusted to $190 \mu \mathrm{l}$ with water, and then $10 \mu \mathrm{l}$ of $30 \% \mathrm{ZnSO}_{4}$ was added for protein precipitation. After $15 \mathrm{~min}$ incubation at room temperature, the samples were centrifuged at $4000 \mathrm{rpm}$ for $5 \mathrm{~min}$, and the supernatants as well as serially diluted NO standards were transferred to microcentrifuge tubes containing $0.5 \mathrm{~g}$ cadmium beads (sequentially prewashed with $\mathrm{H}_{2} \mathrm{O}$, $0.1 \mathrm{M} \mathrm{HCl}$, and $0.1 \mathrm{M} \mathrm{NH}_{4} \mathrm{OH}, \mathrm{pH} 9.6$ ) and incubated at room temperature overnight with agitation to convert nitrate to nitrite. After $5 \mathrm{~min}$ centrifugation at $4000 \mathrm{rpm}, 60 \mu \mathrm{l}$ of supernatant was added to wells in duplicate in a 96-well plate and adjusted to $100 \mu \mathrm{l}$ with water. Greiss reagent $(100 \mu \mathrm{l})$ was then added to each well and incubated for $5 \mathrm{~min}$ at room temperature. The nitrite level in each edema fluid was determined by reading the absorbance at $570 \mathrm{~nm}$ in a microtiter plate reader, and the total NO level of each paw 
a

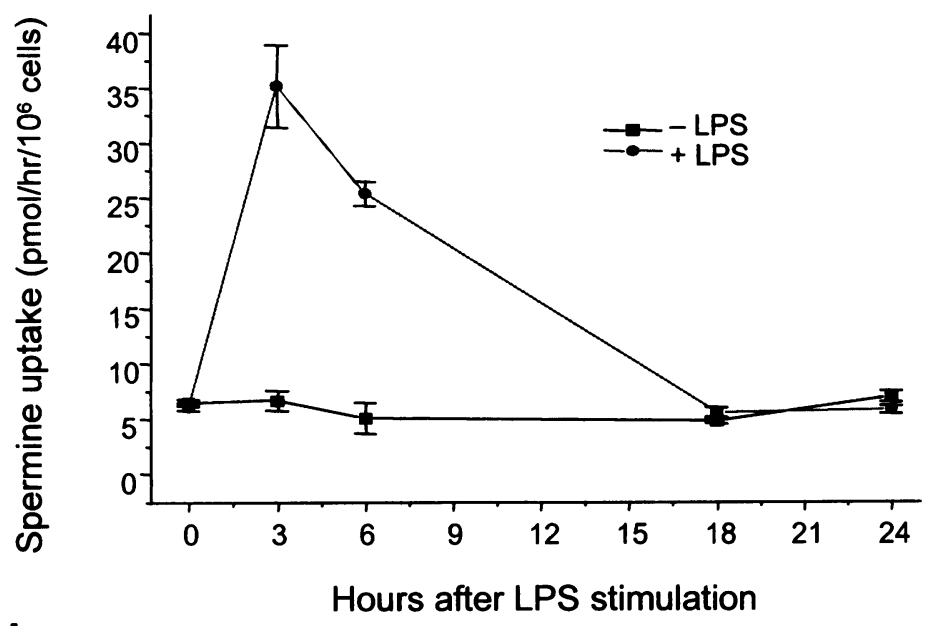

b

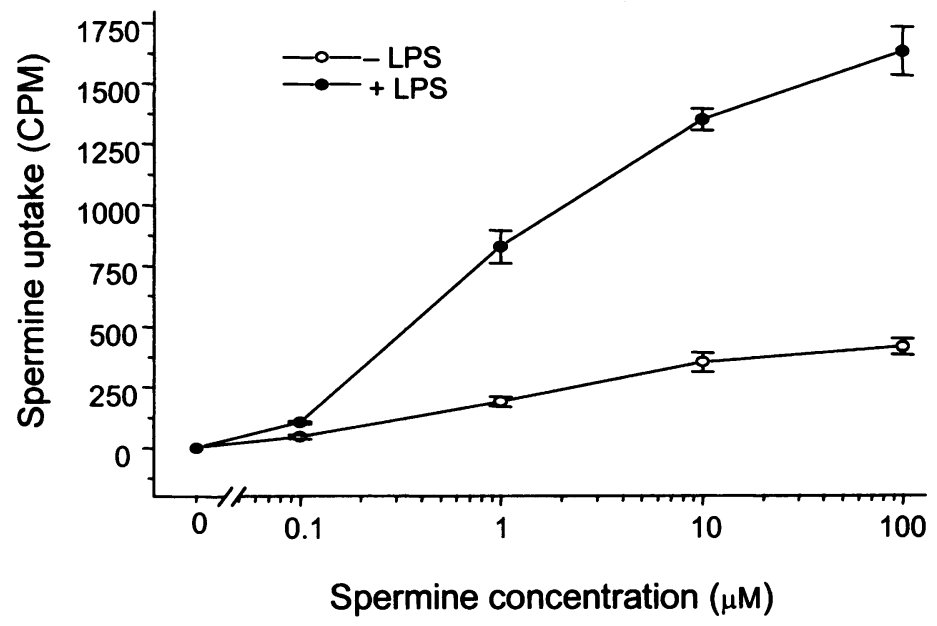

Fig. 1. Spermine uptake in human monocytes is increased by LPS stimulation. Human monocyte cultures were treated with LPS (100 ng/ml) or vehicle PBS for 0 to $24 \mathrm{hr}$ (a) or for $2 \mathrm{hr}$ (b), then incubated with the indicated concentration of spermine with ${ }^{14} \mathrm{C}$-labeled spermine tracer at either $4^{\circ} \mathrm{C}$ or $37^{\circ} \mathrm{C}$ for $25 \mathrm{~min}$. Cell-associated radioactivity (CPM) was measured on a Packard microplate scintillation counter, and spermine uptake (CPM) is expressed as the difference between cell-associated radioactivity at $37^{\circ} \mathrm{C}$ and that at $4^{\circ} \mathrm{C}$ at each spermine concentration. Results are the mean of spermine uptake \pm SE. was calculated as $X(\mathrm{nmol} / \mathrm{paw})=$ nitrite/nitrate in the sample $(\mathrm{nmol} / \mathrm{ml}) \times$ paw edema volume (ml).

\section{Results}

Stimulation of Human Monocytes with LPS Increases Polyamine Uptake

Preliminary experiments established that uptake of spermine, spermidine, and putrescine in monocyte cultures was linear over a 60 -min period at $37^{\circ} \mathrm{C}$; this accumulation was not observed at $4^{\circ} \mathrm{C}$ (not shown). The component of uptake at $4^{\circ} \mathrm{C}$, reflecting binding or passive diffusion, was subtracted from the total uptake at $37^{\circ} \mathrm{C}$, and this net amount was defined as specific polyamine uptake. In subsequent experiments, the accumulation of radiolabeled polyamines in monocyte cultures was measured over a 25-min period. LPS induced a significant increase in spermine uptake compared to unstimulated, quiescent monocyte culture controls. As shown in Figure la, peak spermine uptake was observed 3 hr after exposure of monocyte cultures to LPS; by $18 \mathrm{hr}$, spermine accumulation declined to basal (unstimulated) rates. We next measured the effects of LPS on monocyte uptake of other polyamines (spermidine and putrescine) and observed a similar time course for LPS-induced uptake of these polyamines (not shown). Addition of spermine $(10 \mu \mathrm{M})$ completely inhibited the uptake of spermidine $(35 \mu \mathrm{M})$ and putrescine ( $35 \mu \mathrm{M}$ ), indicating that human monocytes express the nonselective polyamine transporter, which is sensitive to LPS stimulation.

Spermine uptake exhibited saturation kinetics as the concentration of spermine in the media 
was increased (Fig. 1b). Wilkinson analysis of these concentration-rate data using PC Enzpack software indicated that the $V_{\max }$ and $K_{\mathrm{m}}$ values describing the uptake of spermine in unstimulated human monocyte cultures were $54.6 \pm$ $1.71 \mathrm{pmol} / 25 \mathrm{~min} / 10^{6}$ cells and $1.15 \pm 0.185$ $\mu \mathrm{M}$, respectively. LPS stimulation significantly increased the $V_{\max }$ value of spermine uptake to $213 \pm 9.57 \mathrm{pmol} / 25 \mathrm{~min} / 10^{6}$ cells, but the $K_{\mathrm{m}}$ value $(1.00 \pm 0.235 \mu \mathrm{M})$ was not significantly changed by LPS. Eadie-Hofstee plot analysis of spermine uptake was linear in both unstimulated and LPS-stimulated monocytes, suggesting that spermine interacted with a single transporter system.

\section{Inhibition of Monocyte Polyamine Uptake by BAP}

On the basis of our earlier observations that spermine deactivates macrophages (17), we considered it plausible that increased spermine uptake following monocyte activation might serve as a mechanism to prevent excessive macrophage activity. Our approach to study this hypothesis was to identify an inhibitor of spermine uptake in LPS-stimulated monocytes; the effects of this inhibitor on monocyte activation in vitro and monocyte-mediated tissue responses in vivo could then be examined. Accordingly, we screened a panel of spermine analogs for inhibition of spermine uptake in LPS-stimulated human monocyte cultures. One piperazine-like spermine analog, 1,4bis(3-aminopropyl)-piperazine (BAP), decreased monocyte spermine accumulation in a dose-dependent manner; the $50 \%$ inhibitory concentration $\left(\mathrm{IC}_{50}\right)$ of BAP was $7 \pm 2.8 \mu \mathrm{M}$ in the presence of $35 \mu \mathrm{M}$ spermine (Fig. 2a). BAP inhibition of spermine uptake was overcome by sufficiently high concentrations of spermine (Fig. 2b), giving direct evidence that BAP is a competitive inhibitor of monocyte spermine uptake. The inhibitory effect of BAP on spermine uptake was not due to cytotoxicity, because up to $1 \mathrm{mM}$ of BAP did not suppress thiazoyl blue (MTT) metabolism in human monocytes (not shown). BAP also inhibited the uptake of spermidine and putrescine, but it did not inhibit the uptake of glutamine or aspartic acid in monocytes (Fig. 2c). Thus, BAP is a specific, competitive inhibitor of the LPS-inducible polyamine accumulation in human monocytes.

\section{Inhibition of Spermine Uptake Restores Monocyte TNF Synthesis}

We next measured TNF release from human monocytes in cultures supplemented with LPS and BAP. Addition of BAP alone did not activate monocytes to release TNF (Fig. 3); we also observed no significant effect of BAP on the magnitude of LPS-induced TNF release (not shown). BAP treatment, however, fully restored TNF synthesis in spermine-suppressed monocytes and the $50 \%$ effective concentration that restored TNF release in spermine treated cultures $\left(\mathrm{EC}_{50}=\right.$ $8 \pm 3 \mu \mathrm{M}$ ) was comparable to the $\mathrm{IC}_{50}$ of $\mathrm{BAP}$ as an inhibitor of spermine uptake (Fig. 3). The TNF-enhancing activity of BAP in the presence of spermine was significantly correlated with the inhibitory activity of BAP against monocyte spermine uptake (correlation coefficient $=0.99$; $p<0.05)$. Putrescine shares the nonselective polyamine transporter with spermine but does not exert an inhibitory activity on monocyte cytokine synthesis (17). Given the requirement of spermine uptake for its inhibitory activity on monocyte TNF production, we reasoned that putrescine might also enhance cytokine production in spermine-suppressed human monocytes by competitively inhibiting the uptake of spermine. We therefore measured TNF release of sperminetreated human monocytes in the presence or absence of putrescine and observed a significant increase of TNF release by putrescine treatment in spermine-suppressed monocytes (not shown). Thus, with respect to inhibition of macrophage activation, spermine uptake is required for spermine inhibitory activity and increased spermine uptake in activated monocytes is a mechanism that acts to prevent monocyte overactivation.

\section{Inhibition of Spermine Uptake Enhances Carrageenan-Induced Inflammation In Vivo}

It has been well documented that spermine levels are increased in various inflammatory states including infection $(16,22,23)$, tissue injury $(14,24,25)$, and cancer $(15,26)$, suggesting a role of endogenous spermine in regulating the inflammatory process. We recently demonstrated that direct application of exogenous spermine into animal footpad suppresses carrageenan-induced paw edema (17), an inflammatory response that is predominantly mediated by macrophage products (e.g., TNF and NO) (27-29). While these results suggest an anti-inflammatory activity of spermine, it was previously unknown 
a

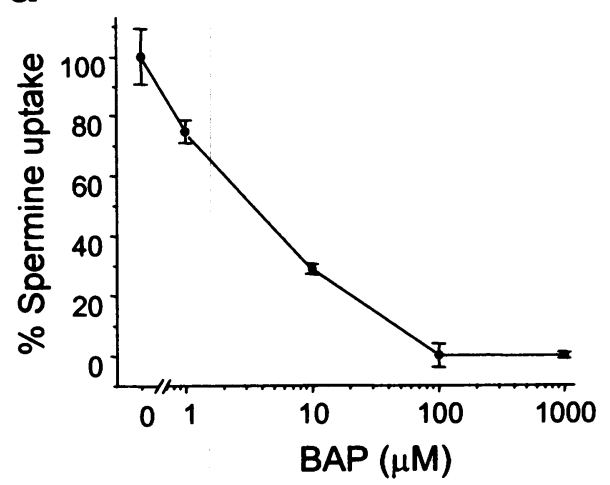

C
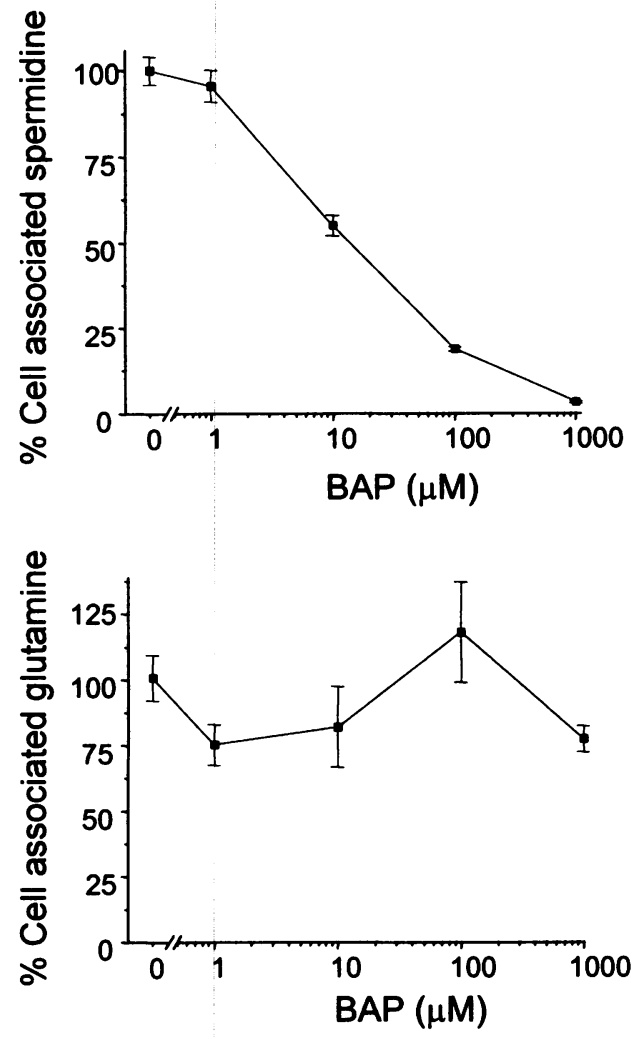

Fig. 2. BAP is a specific, competitive inhibitor of the nonselective polyamine transporter in human monocyte cultures. (a) BAP inhibits spermine uptake in LPS-stimulated human monocytes. Human monocytes were stimulated by LPS (100 $\mathrm{ng} / \mathrm{ml})$ for $2 \mathrm{hr}$, then treated with BAP in the concentrations indicated for 30-60 $\mathrm{min}$ followed by 25 min incubation with $35 \mu \mathrm{M}$ spermine with ${ }^{14} \mathrm{C}$ labeled spermine tracer at either $4^{\circ} \mathrm{C}$ or $37^{\circ} \mathrm{C}$.

Spermine uptake (CPM) is defined by the difference between cell-associated radioactivity at $37^{\circ} \mathrm{C}$ and that at $4^{\circ} \mathrm{C}$, and expressed as percentage of the control (no BAP) cultures. Results are percentage of control mean \pm SE. (b) Competitive inhibition of spermine uptake by BAP. Human monocytes were stimulated by LPS (100 ng/ml) for $2 \mathrm{hr}$, then incu-
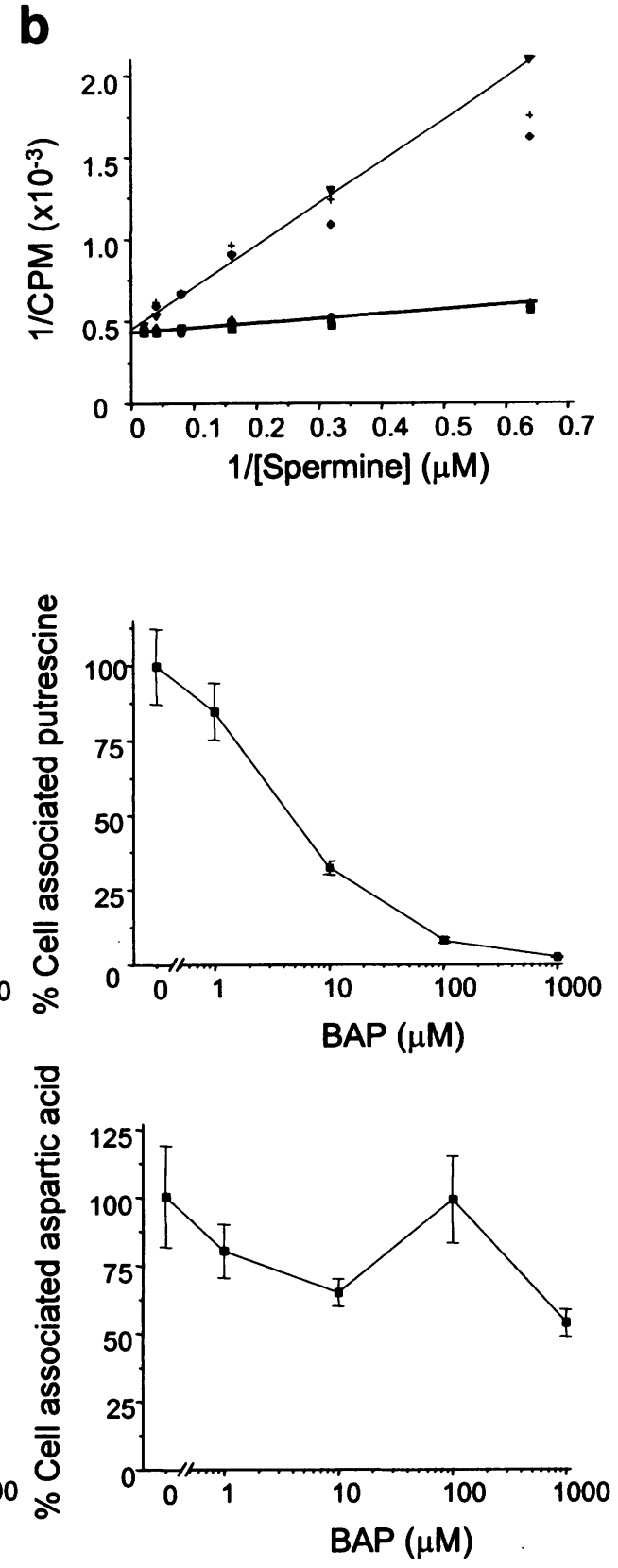

bated with the indicated concentrations of spermine with ${ }^{14} \mathrm{C}$-labeled spermine tracer at either $4^{\circ} \mathrm{C}$ or $37^{\circ} \mathrm{C}$ for $25 \mathrm{~min}$ in the absence or presence of 500 $\mu \mathrm{M}$ of BAP. Cell-associated radioactivity (CPM) was then measured, and spermine uptake (CPM) in human monocytes is shown as a reciprocal plot. The heavy line denotes spermine uptake in the absence of BAP, and the thin line, in the presence of BAP. (c) BAP inhibition of polyamine uptake is specific. Human monocytes were stimulated by LPS (100 $\mathrm{ng} /$ $\mathrm{ml}$ ) for $2 \mathrm{hr}$, then incubated individually with ${ }^{14} \mathrm{C}$ labeled spermine, putrescine, glutamine, or aspartic acid at $37^{\circ} \mathrm{C}$ for $25 \mathrm{~min}$ in the absence or presence of BAP at the concentrations indicated. Cell-associated radioactivity (CPM) was measured and presented as percentage of control (no BAP) \pm SE. 


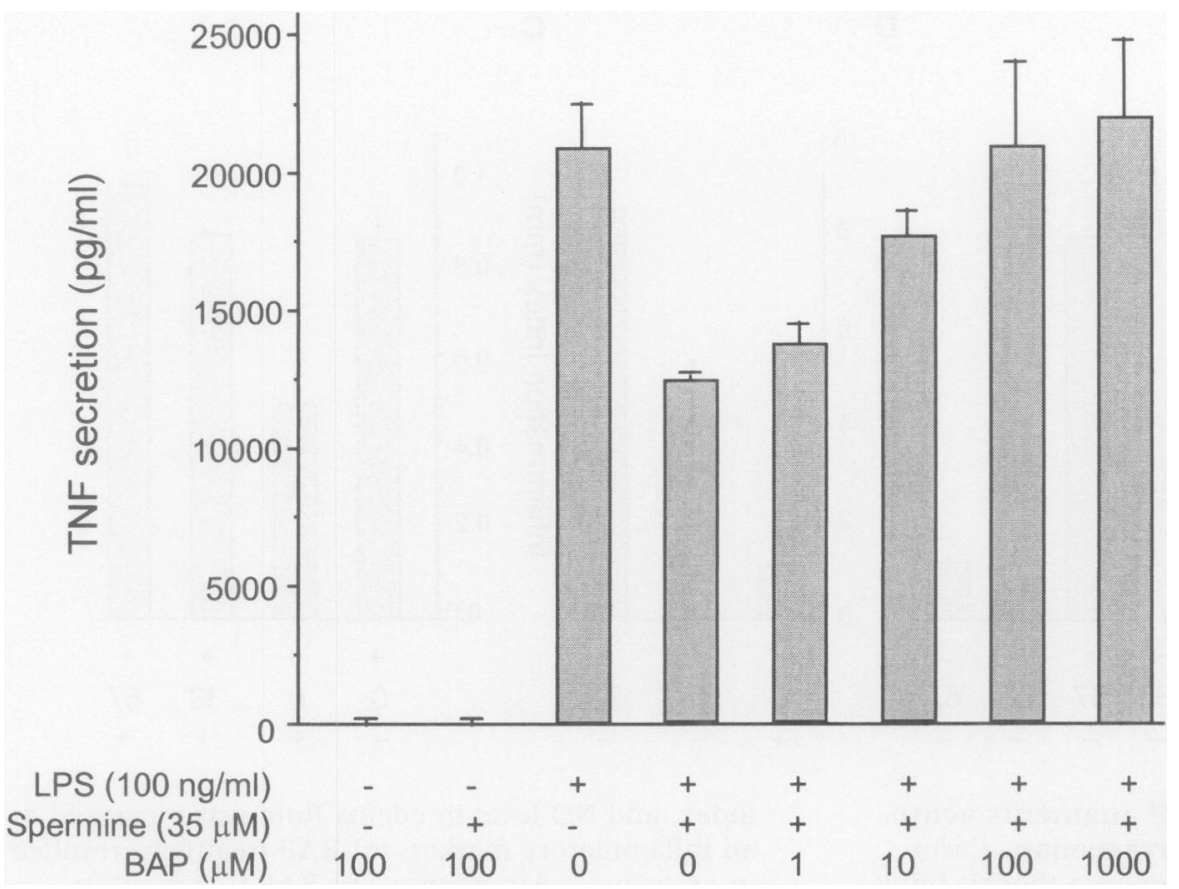

Fig. 3. Blockade of spermine uptake with BAP restores sperminesuppressed TNF synthesis. Human monocytes were pretreated with spermine in the absence or presence of BAP at the indicated concentrations for 30-60 min, then stimulated by LPS (100 ng/ ml) for $4 \mathrm{hr}$. Secreted TNF in cell culture supernatants was measured by ELISA. Results presented are the mean of TNF levels $\pm \mathrm{SE}$. whether endogenous spermine normally participates in the control of inflammatory responses. Accordingly, we evaluated the effects of BAP in carrageenan-induced paw edema by subcutaneous injection of BAP and carrageenan. Administration of BAP alone into rat foot pads failed to stimulate the development of edema in the absence of carrageenan, but coadministration of BAP with carrageenan significantly augmented carrageenan-induced foot pad inflamed (Fig. 4a). We also measured the levels of NO (total nitrite/ nitrates) in the inflammatory paws and observed a significant increase of NO production in BAPtreated paws as compared with the controls (Fig. 4b). The direct involvement of spermine in BAP-enhanced excessive inflammation was supported by the observation that BAP treatment fully "restores" exogenous spermine-suppressed inflammatory responses, whereas additional exogenous spermine significantly reduced inflammation (Fig. 4c) typical of BAP treatment (Fig. 4a). Taken together, these results indicate that endogenous spermine can be incorporated into macrophages to restrain the innate inflammatory response.

\section{Discussion}

Four distinct lines of evidence from the present study indicate that human monocytes might use a single nonselective transporter for the uptake of spermine. First, Eadie-Hofstee plot analysis of spermine uptake was linear, suggesting the presence of a single spermine transporter in human monocytes. Second, spermine treatment inhibits the uptake of putrescine and spermidine, indicating that the transporter for spermine is shared by putrescine and spermidine in human monocytes. This is further supported by the observation that putrescine effectively blocks the uptake-dependent effect of spermine on monocyte cytokine synthesis. Third, a similar time course for LPS-stimulated monocyte uptake of spermine, spermidine, and putrescine was observed, suggesting that LPS-stimulated uptake of polyamines is due to the enhancement of a nonselective transporter activity.

The fourth line of evidence is the inhibition of polyamine uptake by the piperazine-like spermine homologue BAP. BAP inhibited the uptake of all three polyamines by a similar magnitude but had no effect on the uptake of structurally unrelated molecules glutamine and aspartic acid. Thus, BAP is a specific inhibitor of the nonselective transporter in human monocytes. It has been well documented that polyamine transport is an integral part of the system that regulates intracellular polyamine levels according to various needs (20).

A role for monocyte polyamine transport activity, however, has not been previously re- 


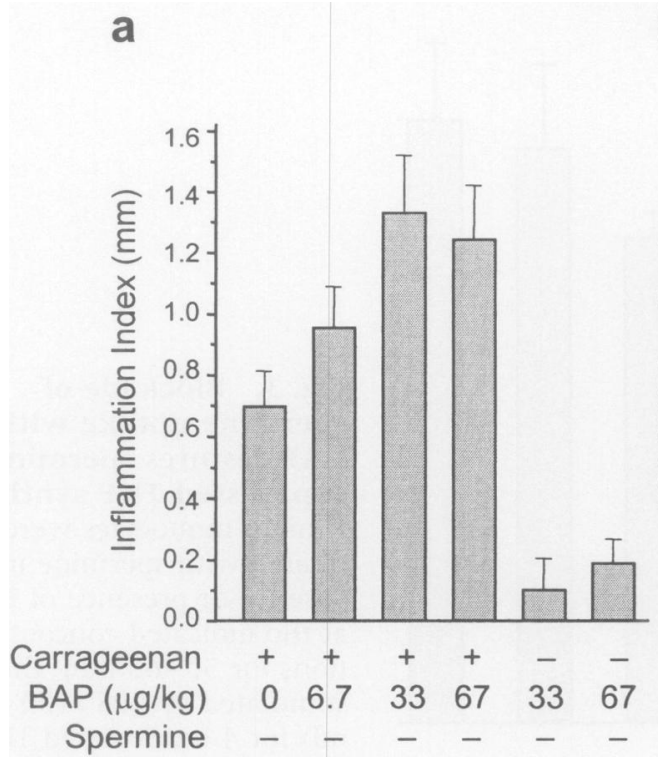

Fig. 4. Administration of BAP augments acute inflammatory responses to carrageenan. Carrageenan $(0.2 \%$ in PBS) was injected into the left hind footpad of male Lewis rats in combination with the indicated concentration of spermine and/or BAP. Vehicle PBS was injected into the right hind footpad as control. Footpad thickness was measured 16-24 $\mathrm{hr}$ later, and the edema fluid was recovered. The difference (in $\mathrm{mm}$ ) between the thickness of left and right hind footpad was defined as the inflammation

ported. An interesting observation in the present study is that monocyte spermine uptake is significantly increased following LPS stimulation. Inhibition of LPS-stimulated spermine uptake by BAP blocks the inhibitory activity of spermine on monocyte TNF production, and it displays a correlation coefficient of $0.99(p<0.05)$ between the inhibitory activity of BAP on spermine uptake and the enhancing activity of BAP on monocyte TNF production. Thus, spermine uptake is required for spermine suppression of monocyte cytokine synthesis, and increased spermine uptake in activated monocytes provides a mechanism to prevent excessive monocyte activation. In agreement with the critical role of spermine in the regulation of monocyte activation, increased spermine uptake in activated monocytes is reversible and time dependent. Thus, monocytes can recover their activity in subsequent inflammatory responses when spermine is reduced or inactivated.

The activation of macrophages is a key event for the initiation and propagation of the innate inflammatory response. In the past, a number of molecules including IL-10, transforming growth b c

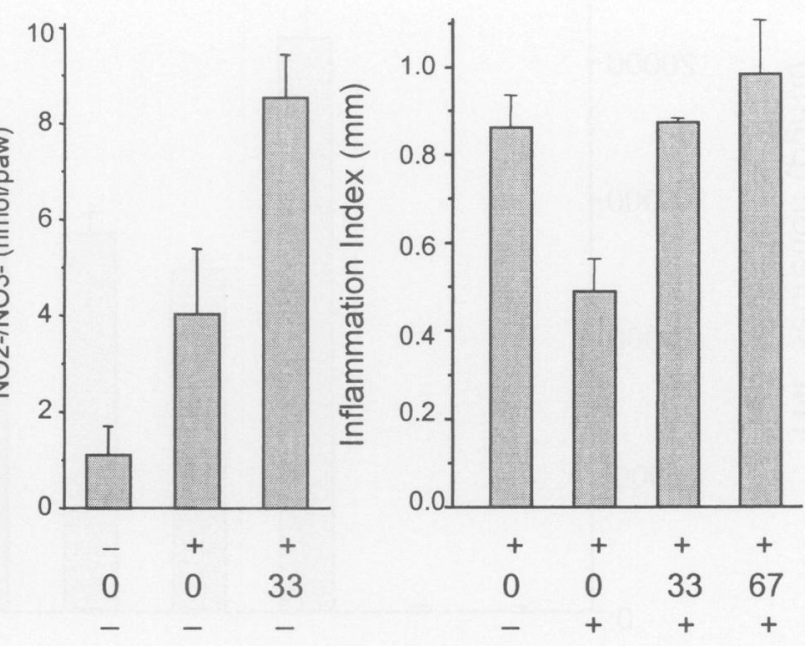

index, and NO level in edema fluid was measured as an inflammatory marker. (a) BAP treatment resulted in excessive inflammation. (b) BAP treatment increased NO production in carrageenan-induced paw edema. (c) The antagonistic interaction between BAP and spermine. Spermine reduces BAP-induced excessive inflammation, whereas the anti-inflammatory activity of exogenous spermine was blocked in the context of inhibition of spermine uptake by BAP treatment.

factor beta (TGF- $\beta$ ), and glucocorticoids have each been implicated in preventing excessive macrophage activation and inflammation $(8-13)$. Unlike IL-10, TGF- $\beta$, or the glucocorticoids, however, spermine is unique in being released locally from dying and injured cells (24). The quantity of spermine released at inflammatory sites will not only reflect the severity of ongoing tissue damage but also proportionally attenuate the innate inflammatory response (Fig. 5). This provides a critical restraining influence on inflammation, as the monocyte-deactivating effects of spermine prevent further release of TNF and NO, thereby limiting the extent of inflammation and associated collateral damage. In agreement with this proposed mechanism, mucosal polyamine deficiency has been implicated in the pathogenesis of inflammatory bowel disease (30).

Some 40 years ago, Hirsch and Dubos discovered increased local spermine levels at the site of mycobacteria infection (16). Subsequent studies in humans and animals indicate that spermine level is also increased in trauma $(14,24,25)$, cancer $(15,26)$, and other infections $(22,23)$. It is 


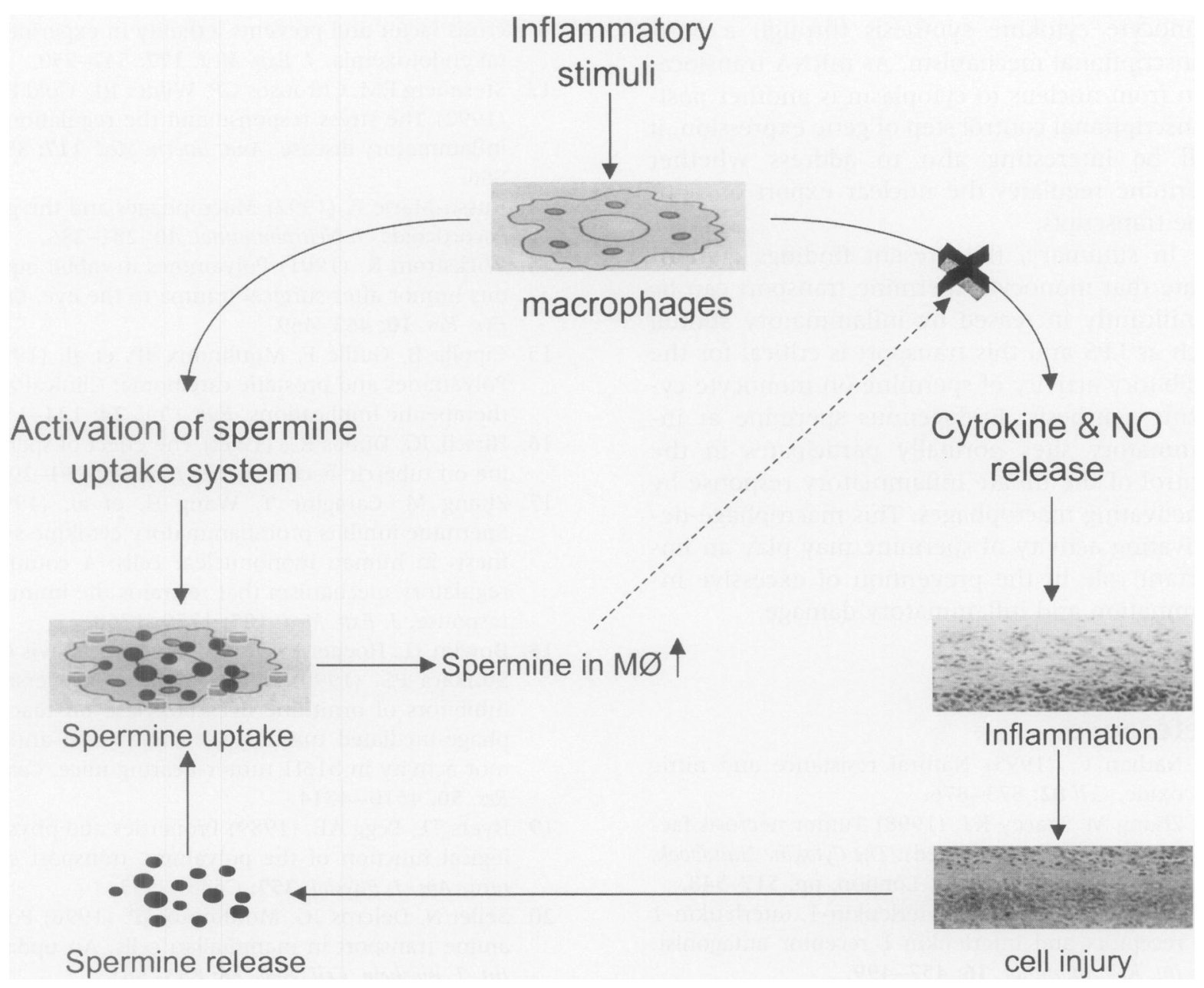

Fig. 5. Regulation of macrophage activation and inflammation by spermine. In response to infection, injury, and other inflammatory stimuli, host macrophages produce various factors, such as TNF, IL-1, and NO, that are critical for an effective immune and inflammatory response but can also be harmful or even lethal to the host at high levels. In response to inflammatory stimuli, the activity of the

interesting that the innate immune response is generally defective in these inflammatory conditions (31-33). Considering the anti-inflammatory activity of endogenous spermine, it is reasonable to consider that spermine accumulation in macrophages and monocytes may contribute to this immune dysfunction. It will be interesting to test whether the blockade of spermine activity with spermine antagonists like BAP can restore the innate immune response in these conditions.

The data presented in this work indicate that cellular uptake of spermine is required for the inhibitory effect of spermine on monocyte cytokine synthesis. Recent evidence (34) indicates that fetuin, a negative acute-phase protein, is spermine uptake system in activated macrophages is increased, leading to the incorporation of spermine from the surrounding environment. Spermine accumulation at inflammatory sites enhances intracellular macrophage uptake of spermine, resulting in the down-regulation of macrophage activation and inflammation.

required for spermine inhibition on monocyte cytokine synthesis, because anti-fetuin antibodies prevent the cytokine-inhibiting effects of spermine. The relation between fetuin and the monocyte uptake of spermine is unknown. Immunoelectron microscopic studies (35) have revealed that intracellular spermine is predominately located on ribosomes of the rough endoplasmic reticulum, and it is one of the components of biologically active ribosomes. It is therefore possible that spermine might suppress monocyte cytokine synthesis by regulating monocyte cytokine mRNA translation or mRNA stabilization. In agreement with this, our previous studies (17) show that spermine suppresses 
monocyte cytokine synthesis through a posttranscriptional mechanism. As mRNA translocation from nucleus to cytoplasm is another posttranscriptional control step of gene expression, it will be interesting also to address whether spermine regulates the nuclear export of cytokine transcripts.

In summary, the present findings demonstrate that monocyte spermine transport can be significantly increased by inflammatory stimuli such as LPS and this transport is critical for the inhibitory activity of spermine on monocyte cytokine synthesis. Endogenous spermine at inflammatory sites normally participates in the control of the innate inflammatory response by deactivating macrophages. This macrophage-deactivating activity of spermine may play an important role in the prevention of excessive inflammation and inflammatory damage.

\section{References}

1. Nathan C. (1995) Natural resistance and nitric oxide. Cell 82: 873-876.

2. Zhang M, Tracey KJ. (1998) Tumor necrosis factor. In: Thomson AW (ed). The Cytokine Handbook, 3rd ed. Academic Press, London, pp. 517-548.

3. Dinarello CA. (1998) Interleukin-1, interleukin-1 receptors and interleukin-1 receptor antagonist. Int. Rev. Immunol. 16: 457-499.

4. Pfeffer K, Matsuyama T, Kundig TM, et al. (1993) Mice deficient for the $55 \mathrm{kD}$ tumor necrosis factor receptor are resistant to endotoxic shock, yet succumb to I. monocytogenes infection. Cell 73: 457467.

5. Fenton MJ, Golenbock DT. (1998) LPS-binding proteins and receptors. J. Leukoc. Biol. 64: 25-32.

6. Glauser MP. (1996) The inflammatory cytokines. New developments in the pathophysiology and treatment of septic shock. Drugs 52 Suppl 2: 9-17.

7. Tracey KJ, Fong Y, Hesse DG. (1986) Shock and tissue injury induced by recombinant human cachectin. Science 234: 470-474.

8. Tsunawaki S, Sporn M, Ding A, Nathan C. (1988) Deactivation of macrophages by transforming growth factor-beta. Nature 334: 260-262.

9. Kulkarni AB, Huh CG, Becker D, et al. (1993) Transforming growth factor beta 1 null mutation in mice causes excessive inflammatory response and early death. Proc. Natl. Acad. Sci. U.S.A. 90: 770-774.

10. Howard M, Muchamuel T, Andrade S, Menon S. (1993) Interleukin 10 protects mice from lethal endotoxemia. J. Exp. Med. 177: 1205-1208.

11. Gerard C, Bruyns C, Marchant A, et al. (1993) Interleukin 10 reduces the release of tumor ne- crosis factor and prevents lethality in experimental endotoxemia. J. Exp. Med. 177: 547-550.

12. Sternberg EM, Chrousos GP, Wilder RL, Gold PW. (1992) The stress response and the regulation of inflammatory disease. Ann. Intern. Med. 117: 854866.

13. Russo-Marie F. (1992) Macrophages and the glucocorticoids. J. Neuroimmunol. 40: 281-286.

14. Wickstrom K. (1991) Polyamines in rabbit aqueous humor after surgical trauma to the eye. Curr. Eye. Res. 10: 463-469.

15. Cipolla B, Guille F, Moulinoux JP, et al. (1993) Polyamines and prostatic carcinoma: Clinical and therapeutic implications. Eur. Urol. 24: 124-131.

16. Hirsch JG, Dubos RJ. (1952) The effect of spermine on tubercle bacilli. J. Exp. Med. 95: 191-208.

17. Zhang $M$, Caragine $T$, Wang $H$, et al. (1997) Spermine inhibits proinflammatory cytokine synthesis in human mononuclear cells: A counterregulatory mechanism that restrains the immune response. J. Exp. Med. 185: 1759-1768.

18. Bowlin TL, Hoeper BJ, Rosenberger AL, Davis GF, Sunkara PS. (1990) Effects of three irreversible inhibitors of ornithine decarboxylase on macrophage-mediated tumoricidal activity and antitumor activity in bl6fl tumor-bearing mice. Cancer Res. 50: $4510-4514$.

19. Byers TL, Pegg AE. (1989) Properties and physiological function of the polyamine transport system. Am. J. Physiol. 257: C545-C553.

20. Seiler N, Delcros JG, Moulinoux JP. (1996) Polyamine transport in mammalian cells. An update. Int. J. Biochem. Cell. Biol. 28: 843-861.

21. Haven CA, Olson JW, Arcot SS, Gillespie MN. (1992) Polyamine transport and ornithine decarboxylase activity in hypoxic pulmonary artery smooth muscle cells. Am. J. Respir. Cell Mol. Biol. 7: 286-292.

22. Colombatto S, De Agostini M, Corsi D, Sinicco A. (1989) Polyamines in lymphocytes from patients infected by human immunodeficiency virus. Biol. Chem. Hoppe Seyler 370: 745-748.

23. Clarke JR, Tyms AS. (1991) Polyamine biosynthesis in cells infected with different clinical isolates of human cytomegalovirus. J. Med. Virol. 34: 212-216.

24. Gaugas JM. (1980) Polyamines in biomedical research. In: Gaugas JM (ed). Polyamines in Biomedical Research. John Wiley \& Sons, Chichester.

25. Paschen W, Cleef $M$, Rohn G, Muller $M$, Pajunen AE. (1993) Ischemia-induced disturbances of polyamine synthesis. Prog. Brain Res. 96: 147-160.

26. Colella G, Cianciulli S, De Santis A, et al. (1994) The determination of polyamines in tumors of the maxillofacial area (in Italian). Minerva Stomatol. 43: 133-136.

27. Wei XQ, Charles IG, Smith A, et al. (1995) Altered immune responses in mice lacking inducible nitric oxide synthase. Nature 375: 408-411.

28. Salvemini D, Wang ZQ, Wyatt PS, et al. (1996) Nitric oxide: A key mediator in the early and late 
phase of carrageenan-induced rat paw inflammation. Br. J. Pharmacol. 118: 829-838.

29. Cunha FQ, Poole S, Lorenzetti BB, Ferreira SH. (1992) The pivotal role of tumour necrosis factor alpha in the development of inflammatory hyperalgesia. Br. J. Pharmacol. 107: 660-664.

30. Kanof ME, Rance NE, Hamilton SR, Luk GD, Lake AM. (1987) Congenital diarrhea with intestinal inflammation and epithelial immaturity. J. Pediatr. Gastroenterol. Nutr. 6: 141-146.

31. Faist E, Schinkel C, Zimmer S. (1996) Update on the mechanisms of immune suppression of injury and immune modulation. World J. Surg. 20: 454-459.
32. Elgert KD, Alleva DG, Mullins DW. (1998) Tumor-induced immune dysfunction: The macrophage connection. J. Leukoc. Biol. 64: 275-290.

33. Taylor KA. (1998) Immune responses of cattle to african trypanosomes: Protective or pathogenic? Int. J. Parasitol. 28: 219-240.

34. Wang $\mathrm{H}$, Zhang $M$, Soda K, Sama A, Tracey KJ. (1997) Fetuin protects the fetus from TNF [letter]. Lancet 350: 861-862.

35. Fujiwara K, Bai G, Kitagawa T, Tsuru D. (1998) Immunoelectron microscopic study for polyamines. J. Histochem. Cytochem. 46: 1321-1328. 